\title{
ON THE PROGENITOR SYSTEM OF THE TYPE Iax SUPERNOVA 2014dt IN M61
}

\author{
Ryan J. Foley ${ }^{1,2}$, Schuyler D. Van Dyk ${ }^{3}$, Saurabh W. Jha ${ }^{4}$, Kelsey I. Clubb ${ }^{5}$, Alexei V. Filippenko ${ }^{5}$, \\ Jon C. Mauerhan ${ }^{5}$, Adam A. Miller ${ }^{6,7,9}$, and Nathan Smith $^{8}$ \\ ${ }^{1}$ Astronomy Department, University of Illinois at Urbana-Champaign, 1002 West Green Street, Urbana, IL 61801, USA \\ ${ }^{2}$ Department of Physics, University of Illinois at Urbana-Champaign, 1110 West Green Street, Urbana, IL 61801, USA \\ ${ }^{3}$ IPAC/Caltech, Mail Code 100-22, Pasadena, CA 91125, USA \\ ${ }^{4}$ Department of Physics and Astronomy, Rutgers, The State University of New Jersey, 136 Frelinghuysen Road, Piscataway, NJ 08854, USA \\ ${ }^{5}$ Department of Astronomy, University of California, Berkeley, CA 94720-3411, USA \\ ${ }^{6}$ Jet Propulsion Laboratory, California Institute of Technology, 4800 Oak Grove Drive, MS 169-506, Pasadena, CA 91109, USA \\ ${ }^{7}$ California Institute of Technology, Pasadena, CA 91125, USA \\ ${ }^{8}$ Steward Observatory, University of Arizona, Tucson, AZ 85721, USA \\ Received 2014 December 1; accepted 2014 December 16; published 2014 December 31
}

\begin{abstract}
We present pre-explosion and post-explosion Hubble Space Telescope images of the Type Iax supernova (SN Iax) 2014dt in M61. After astrometrically aligning these images, we do not detect any stellar sources at the position of the SN in the pre-explosion images to relatively deep limits ( $3 \sigma$ limits of $M_{\mathrm{F} 438 \mathrm{~W}}>-5.0 \mathrm{mag}$ and $M_{\mathrm{F} 814 \mathrm{~W}}>-5.9 \mathrm{mag}$ ). These limits are similar to the luminosity of SN 2012Z's progenitor system $\left(M_{\mathrm{F} 435 \mathrm{~W}}=-5.43 \pm 0.15\right.$ and $\left.M_{\mathrm{F} 814 \mathrm{~W}}=-5.24 \pm 0.16 \mathrm{mag}\right)$, the only probable detected progenitor system in pre-explosion images of a SN Iax, and indeed, of any white-dwarf supernova. SN 2014dt is consistent with having a C/O white-dwarf primary/helium-star companion progenitor system, as was suggested for SN 2012Z, although perhaps with a slightly smaller or hotter donor. The data are also consistent with SN 2014dt having a low-mass red giant or main-sequence star companion. The data rule out main-sequence stars with $M_{\text {init }} \gtrsim 16 M_{\odot}$ and most evolved stars with $M_{\text {init }} \gtrsim 8 M_{\odot}$ as being the progenitor of SN 2014dt. Hot Wolf-Rayet stars are also allowed, but the lack of nearby bright sources makes this scenario unlikely. Because of its proximity $(D=12 \mathrm{Mpc})$, SN 2014dt is ideal for long-term monitoring, where images in $\sim 2 \mathrm{yr}$ may detect the companion star or the luminous bound remnant of the progenitor white dwarf.
\end{abstract}

Key words: galaxies: individual (M61) - supernovae: general - supernovae: individual (SN 2014dt)

\section{INTRODUCTION}

Type Iax supernovae (SNe Iax) are a recently defined class of stellar explosion (Foley et al. 2013, hereafter F13). They are, in many ways, observationally similar to SNe Ia, having comparable spectra and thus compositions (e.g., Li et al. 2003; Branch et al. 2005; Chornock et al. 2006; Jha et al. 2006; Phillips et al. 2007; Foley et al. 2009, 2010a). However, SNe Iax are less energetic, with ejecta velocities near maximum light 20\%-80\% that of typical SNe Ia (e.g., Narayan et al. 2011, F13; White et al. 2014). SNe Iax also tend to have lower luminosity than SNe Ia (e.g., McClelland et al. 2010; Stritzinger et al. 2014a, 2014b), further indicating a low-energy explosion.

One important difference between SNe Iax and SNe Ia is that we have imaged the probable progenitor system of a SN Iax (McCully et al. 2014a, hereafter, M14a), while no progenitor system of a SN Ia has yet been directly observed. For SN 2012Z, M14a detected a luminous, blue source in preexplosion Hubble Space Telescope (HST) images at the SN position. Their favored interpretation is that this source is the nondegenerate He companion star to a $\mathrm{C} / \mathrm{O}$ white dwarf (WD), as originally predicted by $\mathrm{F} 13$ as the likely progenitor scenario for SNe Iax. However, it is also possible that the light came from a massive star that exploded to cause SN 2012 Z or an accretion disk around the exploding WD.

An important prediction from both simple energetic arguments (Foley 2008; Foley et al. 2009, 2013; McCully et al. 2014b) and from detailed explosion models (Jordan et al. 2012; Kromer et al. 2013; Fink et al. 2014) is that at least some of the time, a SN Iax, if coming from a C/O WD, should leave

\footnotetext{
9 Hubble Fellow.
}

behind a bound remnant star. Since the remnant retains a significant amount of radioactive material after the explosion (Kromer et al. 2013), this star will likely become quite luminous on the timescale of years to decades (L. Bildsten et al., in preparation).

Using HST images that include the location of SN 2008ha, a low-luminosity SN Iax (Foley et al. 2009, 2010a; Valenti et al. 2009), from $4 \mathrm{yr}$ after the explosion, Foley et al. (2014) detected a luminous, red source at the position of the SN. Since these images came after the explosion, the source could be a thermally pulsating asymptotic giant branch companion star or a bound remnant star. If the sources detected at the positions of SNe 2008ha and 2012Z are both companion stars, SNe Iax must have a diverse set of progenitor systems.

Until recently, SN 2008ge was the only other SN Iax with pre-explosion HST imaging (Foley et al. 2010b). These data are not particularly deep ( $3 \sigma$ limit of $M_{V}>-6.9 \mathrm{mag}$ ), but rule out particularly massive stars as potential progenitors. Notably, SN 2008ge occurred in an S0 galaxy with no star formation to deep limits (Foley et al. 2010b), which indirectly disfavors a massive star progenitor.

In this Letter, we present deep pre- and post-explosion $H S T$ images of SN 2014dt, a SN Iax discovered in M61 at a distance of only $12.3 \mathrm{Mpc}$. By comparing the pre- and post-explosion images, we precisely determine the position of the SN in the pre-explosion images. We do not detect any star at that position with a $3 \sigma$ limit of $M_{\mathrm{F} 450 \mathrm{~W}}>-5.0 \mathrm{mag}$.

\section{OBSERVATIONS AND DATA REDUCTION}

SN 2014dt was detected in M61 on 2014 October 29.8 (all dates are UT) at $13.6 \mathrm{mag}$ by Nakano \& Itagaki (2014) and promptly classified as a SN Iax by Ochner et al. (2014) from a 


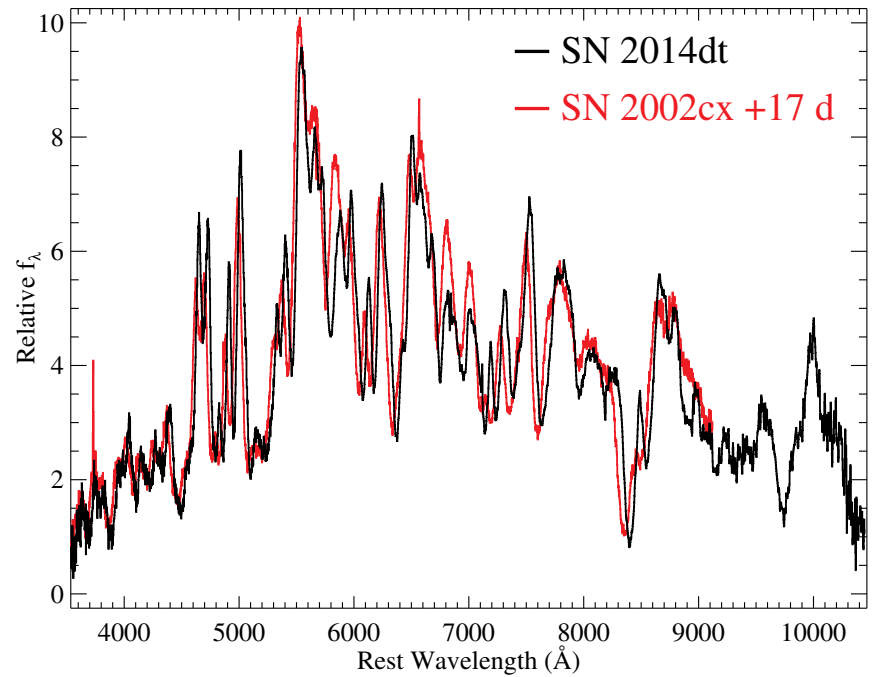

Figure 1. Optical spectrum of SN 2014dt (black) compared to that of SN 2002cx at +17 days (red).

spectrum obtained 2014 October 31.2. The SN was past peak at discovery and there are no recent nondetections which constrain the date of explosion.

M61 is in the Virgo cluster. Using the expanding photosphere method (EPM) applied to SN 2008in, which was hosted by M61, Bose \& Kumar (2014) measured a distance to M61 of $12.3 \mathrm{Mpc}(\mu=30.45 \pm 0.10 \mathrm{mag})$. This distance is consistent with the Tully-Fisher distance of $11.0 \mathrm{Mpc}(\mu=30.21 \pm$ $0.70 \mathrm{mag}$; Schoeniger \& Sofue 1997) and with the redshiftderived distance (corrected for Virgo infall) of $13.1 \mathrm{Mpc}$ $(30.59 \pm 0.16 \mathrm{mag})$. Here we assume the EPM distance modulus, but increase the uncertainty to $0.24 \mathrm{mag}$, corresponding to the offset between the EPM and Tully-Fisher measurements; this range also includes the redshift-derived distance.

On 2014 November 18.6, we obtained a low-resolution spectrum of SN 2014dt with the Kast double spectrograph (Miller \& Stone 1993) on the Shane $3 \mathrm{~m}$ telescope at Lick Observatory. Standard CCD processing and spectrum extraction were accomplished with IRAF. ${ }^{10}$ The data were extracted using the optimal algorithm of Horne (1986). Low-order polynomial fits to calibration-lamp spectra were used to establish the wavelength scale, and small adjustments derived from nightsky lines in the object frames were applied. We employed our own IDL routines to flux calibrate the data and remove telluric lines using the well-exposed continua of spectrophotometric standards (Wade \& Horne 1988; Foley et al. 2003; Silverman et al. 2012). The spectrum is presented in Figure 1.

The position of SN 2014dt was imaged by HST/WFPC2 on 2001 July 27.10 (Program GO-9042; PI Smartt) in F450W (roughly $B$ ) and F814W (roughly I), each for $460 \mathrm{~s}$. We obtained drizzled mosaics from the Hubble Legacy Archive.

We also imaged SN 2014dt with HST/WFC3/UVIS in F438W (roughly $B$ and well matched to the pre-explosion F450W image) on 2014 November 18.89 (Program GO-13683; PI Van Dyk). We took 20 short (20 s) exposures so as to not saturate the SN.

We combined exposures (including cosmic-ray rejection) using AstroDrizzle. We drizzled the images to the native scale of

\footnotetext{
10 IRAF: the Image Reduction and Analysis Facility is distributed by the National Optical Astronomy Observatory, which is operated by the Association of Universities for Research in Astronomy, Inc. (AURA) under cooperative agreement with the National Science Foundation (NSF).
}

WFC3, 0.'04 pixel $^{-1}$. We also ran the individual flt images of the SN through Dolphot, an extension of HSTPhot (Dolphin 2000), and measured the brightness to be $m_{\mathrm{F} 438 \mathrm{~W}}=16.48 \pm 0.01 \mathrm{mag}$.

Using 30 stars in common between the F450W pre-explosion and $\mathrm{F} 438 \mathrm{~W}$ post-explosion images, we computed a relative astrometric solution between the two images and precisely determined the position of SN 2014dt in the pre-explosion image. The position of SN 2014dt has uncertainties of 0.13 and 0.10 pixels $(0.006$ and 0.2005$)$ in the horizontal and vertical directions, respectively. After the initial astrometric match, we are able to transform between the two pre-explosion images with negligible additional positional uncertainty.

Pre- and post-explosion images of SN 2014dt and its surrounding environment are shown in Figure 2.

We conducted Dolphot point-spread function photometry on the individual pre-explosion $H S T$ flt frames, using the suggested Dolphot parameters for WFPC2. We only consider photometry with a "flag" of 0 and an "object type" of 1 (point sources) in our results.

There is no source detected at the position of SN 2014dt in either pre-explosion image. The closest source, detected by Dolphot at 3.6 $\sigma$ with $25.61 \mathrm{mag}$ in F450W only, is 1".64 (97 pc) to the southwest of the SN position. The next closest, detected at $3.6 \sigma$ with $24.60 \mathrm{mag}$ in F814W only, is $33^{\prime \prime} 21$ (190 pc) to the southeast. The closest source detected in both bands (with $24.06 \mathrm{mag}, 11.6 \sigma$ at $\mathrm{F} 450 \mathrm{~W} ; 23.47 \mathrm{mag}, 9.4 \sigma$ at $\mathrm{F} 814 \mathrm{~W}$ ) is 3 .'59 $(210 \mathrm{pc})$ to the northeast. The $3 \sigma$ limiting magnitude corresponds to $m_{\mathrm{F} 450 \mathrm{~W}}>25.7$ and $m_{\mathrm{F} 814 \mathrm{~W}}>24.8 \mathrm{mag}$, respectively.

\section{ANALYSIS}

SN 2014dt is spectroscopically a SN Iax. The spectrum presented in Figure 1 is compared to one of SN 2002cx, the prototypical member of the class (Filippenko 2003; Li et al. 2003), at 17 days after maximum brightness. They are nearly identical except SN 2014dt appears to have a slightly lower ejecta velocity (by $\sim 1500 \mathrm{~km} \mathrm{~s}^{-1}$ ). Importantly, there is no indication of hydrogen in the spectrum.

At $D=12.3 \mathrm{Mpc}$, SN 2014dt had $M \approx-17.1 \mathrm{mag}$ at discovery. The luminosity of SN 2014dt at discovery is similar to that of other SNe Iax (F13) at a similar phase (as determined from its spectrum).

Although it is difficult to measure host-galaxy reddening from SN Iax colors (F13), SN 2014dt does not appear to have any host-galaxy reddening. The $\mathrm{SN}$ has a reasonably blue continuum and there is no $\mathrm{Na} \mathrm{D}$ absorption in our high signal-to-noise ratio low-resolution spectrum to a $3 \sigma$ limit (Leonard \& Filippenko 2001) of $0.1 \AA$. This is consistent with the expected reddening from a relatively clean part of a face-on spiral galaxy.

No sources were detected at the position of SN 2014dt in preexplosion HST images. With our given distance modulus $(\mu=$ $30.45 \mathrm{mag}$ ), and our Milky Way and host-galaxy reddening estimates $(E(B-V)=0.02$ and $0 \mathrm{mag}$, respectively), the $3 \sigma$ limits correspond to $M_{\mathrm{F} 450 \mathrm{~W}}>-5.0$ and $M_{\mathrm{F} 814}>-5.9 \mathrm{mag}$. These limits, converted into blackbody temperature and F814W luminosity limits, are presented in Figure 3. They are similar to the observed brightness of the probable SN 2012Z progenitor system $\left(M_{\mathrm{F} 435 \mathrm{~W}}=-5.43 \pm 0.15\right.$ and $M_{\mathrm{F} 814 \mathrm{~W}}=-5.24 \pm$ $0.16 \mathrm{mag}$ ). Formally, the SN 2014dt limits are 0.40 mag deeper than the SN 2012 Z progenitor system detection in F435W/ F450W. However, this is different by only $1.4 \sigma$ when including uncertainties in the $\mathrm{SN} 2012 \mathrm{Z}$ progenitor-system photometry and the distance to M61. We therefore cannot rule out a 

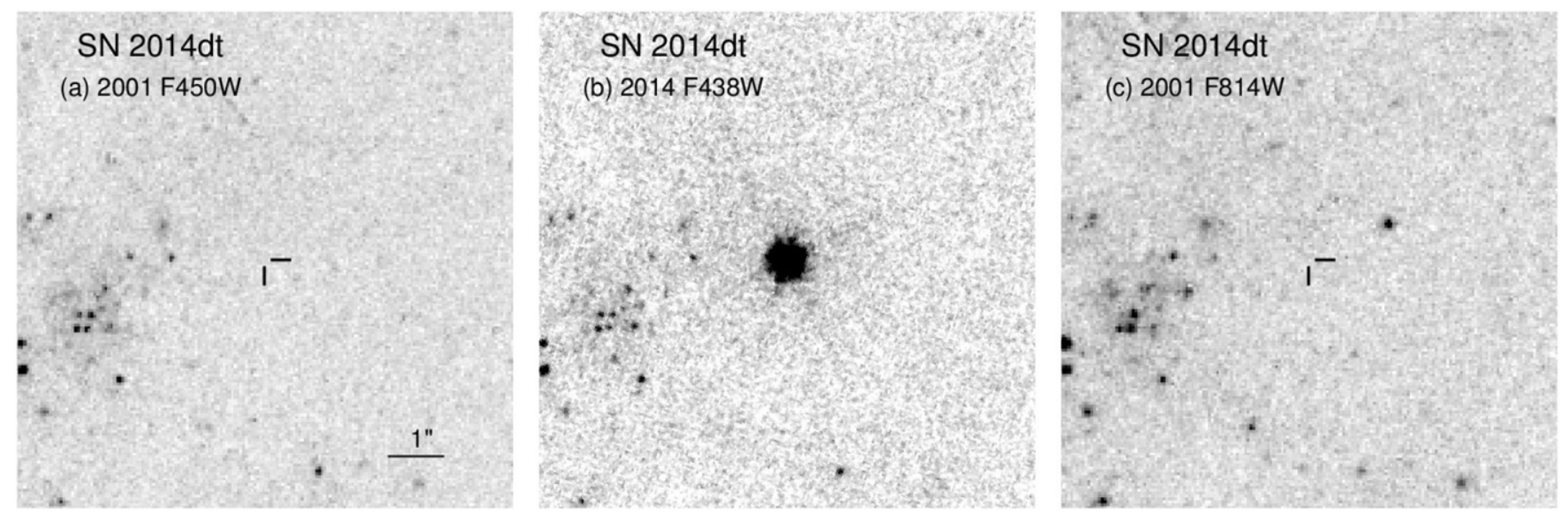

Figure 2. HST/WFC3 F438W image of SN 2014dt (b) and its surrounding environment. Aligned pre-explosion HST/WFPC2 F450W (a) and F814W (c) PC1-chip images are also shown to the same scale. All panels are oriented with north up and east left. The position of SN 2014dt in the pre-explosion images is marked. No star is detected at the SN location in either pre-explosion image.

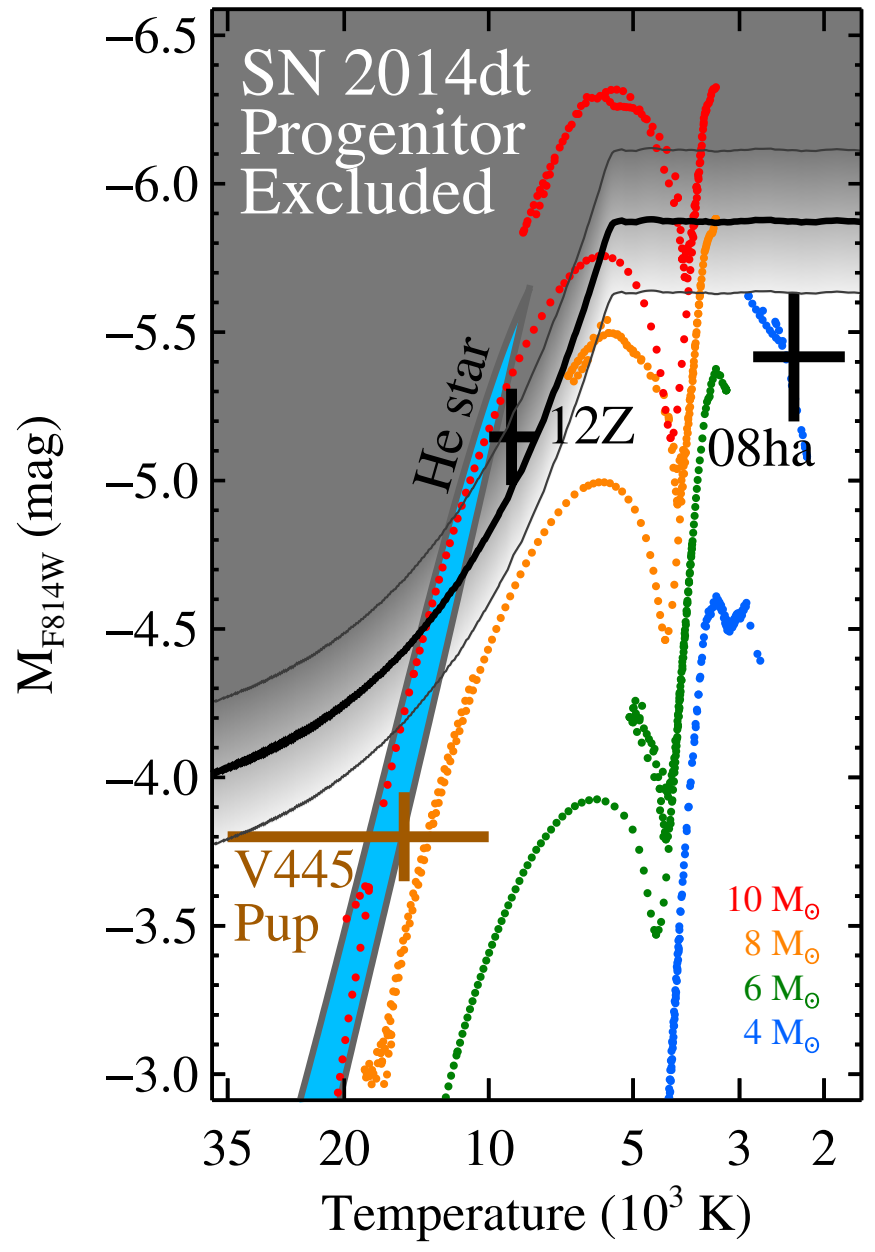

Figure 3. Hertzsprung-Russell diagram for SN Iax progenitor systems. SN 2014dt is not detected, and the region of the H-R diagram excluded is shaded dark gray. The allowed region is white. The black curve represents the single-band $3 \sigma$ limits for blackbody sources. The gray gradient band indicates the additional $1 \sigma$ distance-modulus uncertainty. The SN $2012 \mathrm{Z}$ progenitor system (black cross; M14a) and the V445 Pup progenitor system (brown cross F814W magnitude estimated from temperature and $V$-band magnitude; Woudt et al. 2009) are shown. The source coincident with SN 2008ha in post-explosion images is also marked with a black cross. This source may be the companion star or the bound remnant star (Foley et al. 2014). Also plotted are stellar-evolution tracks for stars with initial masses of 4, 6, 8, and $10 M_{\odot}$ (Bertelli et al. 2009) and the region predicted for one set of He-star progenitor models from Liu et al. (2010, blue region). progenitor system for $\mathrm{SN} 2014 \mathrm{dt}$ that is similar to that of SN $2012 Z$.

Our limits are not quite deep enough to rule out a progenitor system similar to that of the source coincident with SN 2008ha at late times $\left(M_{\mathrm{F} 814 \mathrm{~W}}=-5.42 \pm 0.22 \mathrm{mag}\right)$. Moreover, a system similar to that of V445 Pup (the only known He nova; Kato \& Hachisu 2003), thought to be a C/O WD with a Hestar companion (Kato et al. 2008; Woudt et al. 2009), would be barely undetected in the pre-explosion images. Therefore, SN 2014dt could have a progenitor system similar to any of these comparison objects.

Nonetheless, a large number of potential progenitor systems have been excluded. Using a projected offset of $2.3 \mathrm{kpc}$, and a metallicity map of M61 (Pilyugin et al. 2014), we find a metallicity at the $\mathrm{SN}$ position of $12+\log (\mathrm{O} / \mathrm{H})=8.68$, which on the scale adopted by Pilyugin et al. (2014) is roughly 1.5 times solar. From single-star evolutionary models at this metallicity (Bertelli et al. 2009), the pre-explosion limits are inconsistent with systems containing a red giant (RG) or horizontal branch $(\mathrm{HB})$ star with $M_{\text {init }} \gtrsim 8 M_{\odot}$ or main-sequence stars with $M_{\text {init }} \gtrsim 16 M_{\odot}$. Because of the lack of hydrogen in the SN spectrum, none of these kinds of stars was likely to have been the progenitor anyway; nonetheless, these limits apply to companion stars as well.

The data do not exclude very hot Wolf-Rayet stars, which can be relatively faint at optical wavelengths (Shara et al. 2013). However, Wolf-Rayet stars would likely be physically close to other massive stars. SN 2014dt exploded in a region that is $\sim 100 \mathrm{pc}$ from any detected sources and $\gtrsim 200 \mathrm{pc}$ from any particularly bright sources, making the Wolf-Rayet scenario less likely.

\section{DISCUSSION AND CONCLUSIONS}

Using HST images obtained 13 yr before SN 2014dt, a clear SN Iax, we place limits on its progenitor system. This is the third SN Iax with pre-explosion HST images and the second deepest (after SN 2012Z).

With these data, we are able to exclude many massive stars as progenitors of SN 2014dt. Specifically, main-sequence stars with $M_{\text {init }} \gtrsim 16 M_{\odot}$ and RG/HB stars with $M_{\text {init }} \gtrsim 8 M_{\odot}$ are excluded. Some Wolf-Rayet stars are still allowed, but the lack of bright nearby stars make a Wolf-Rayet progenitor unlikely.

If the progenitor system of SN $2012 Z$ were in M61, we would perhaps marginally detect it. Since we did not detect any sources 
at the position of SN 2014dt, its progenitor system was likely less luminous (in $B$ ) than that of SN $2012 Z$.

Many of the He stars in the He-star-C/O-WD progenitor models of Liu et al. (2010) have roughly the same luminosity but varying temperature. Since the He-star spectral energy distribution peaks in the ultraviolet, the effective temperature dictates the brightness in the HST bands. A large region of the He-star parameter space is still allowed by the current data. However, future data, particularly at shorter ultraviolet wavelengths, should be able to place strong constraints on the existence of such a star.

The pre-explosion data also do not exclude an RG similar to what may be the companion of SN 2008ha, although the data are within $\sim 0.5 \mathrm{mag}$ of being able to reveal such a star. Again, future data should provide a clear detection of a similar star, but as mentioned in Section 1 SN 2008ha, the interpretation of such a detection would be somewhat ambiguous.

There are now three SNe Iax with reasonably deep preexplosion images. A probable luminous blue progenitor system was detected for SN $2012 Z$ (M14a), the SN with the deepest pre-explosion data, while no progenitor system was detected for either SNe 2008ge (Foley et al. 2010b) or 2014dt. Foley et al. (2014) detected a luminous red source coincident with SN 2008ha $4 \mathrm{yr}$ after the explosion that could be a companion star or the bound remnant of the progenitor WD. These four $\mathrm{SNe}$ independently and jointly rule out massive stars as SN Iax progenitors.

SN 2014dt, being at $D=12.3 \mathrm{Mpc}$, is likely the closest SN Iax yet discovered (SN 2010ae was at $12.9 \mathrm{Mpc}$; Stritzinger et al. 2014a), making it a great candidate for long-term monitoring. Of the five SNe Iax discovered within $\sim 20 \mathrm{Mpc}$ (SNe 2008ge, 2008ha, 2010ae, 2010el, and 2014dt), SN 2014dt is the best SN for such observations; SNe 2010ae and 2010el have significant reddening, SN 2008ge was close to a bright galactic nucleus, and SN 2008ha was the faintest and most distant. SNe Iax fade by $\sim 12$ mag in the first two years (McCully et al. 2014b). SN 2014dt peaked at $M \approx-18$ mag, and will be roughly as bright as the pre-explosion image limits in two years. Around that time, deep images may be able to detect emission from either a companion star or the WD remnant.

Based on observations made with the NASA/ESA Hubble Space Telescope, obtained at the Space Telescope Science Institute (STScI), which is operated by the Association of Universities for Research in Astronomy, Inc., under NASA contract NAS 5-26555. These observations are associated with and funded through program GO-13683. Data were obtained through the Hubble Legacy Archive, which is a collaboration between STScI/NASA, the Space Telescope European Coordinating Facility (ST-ECF/ESA), and the Canadian Astronomy Data Centre (CADC/NRC/CSA).
SN Iax research at the University of Illinois is supported in part through NASA/HST grant GO-12999.01. This research at Rutgers University was supported through NASA/HST grant GO-12913.01 and National Science Foundation (NSF) CAREER award AST-0847157 to S.W.J. A.A.M. acknowledges support for this work by NASA from Hubble Fellowship grant HST-HF-51325.01, awarded by STScI. A.V.F.'s group at U.C. Berkeley is supported by the Christopher R. Redlich Fund, the TABASGO Foundation, and NSF grant AST-1211916.

Facility: HST (WFPC2, WFC3), Shane (Kast)

\section{REFERENCES}

Bertelli, G., Nasi, E., Girardi, L., \& Marigo, P. 2009, A\&A, 508, 355 Bose, S., \& Kumar, B. 2014, ApJ, 782, 98

Branch, D., Baron, E., Hall, N., Melakayil, M., \& Parrent, J. 2005, PASP, 117,545

Chornock, R., Filippenko, A. V., Branch, D., et al. 2006, PASP, 118, 722

Dolphin, A. E. 2000, PASP, 112, 1383

Filippenko, A. V. 2003, in From Twilight to Highlight: The Physics of Supernovae ed. W. Hillebrandt \& B. Leibundgut (Berlin: Springer), 171

Fink, M., Kromer, M., Seitenzahl, I. R., et al. 2014, MNRAS, 438, 1762 Foley, R. J. 2008, CBET, 1576, 2

Foley, R. J., Brown, P. J., Rest, A., et al. 2010a, ApJL, 708, L61

Foley, R. J., Challis, P. J., Chornock, R., et al. 2013, ApJ, 767, 57

Foley, R. J., Chornock, R., Filippenko, A. V., et al. 2009, AJ, 138, 376

Foley, R. J., McCully, C., Jha, S. W., et al. 2014, ApJ, 792, 29

Foley, R. J., Papenkova, M. S., Swift, B. J., et al. 2003, PASP, 115, 1220

Foley, R. J., Rest, A., Stritzinger, M., et al. 2010b, AJ, 140, 1321

Horne, K. 1986, PASP, 98, 609

Jha, S., Branch, D., Chornock, R., et al. 2006, AJ, 132, 189

Jordan, G. C., IV, Perets, H. B., Fisher, R. T., \& van Rossum, D. R. 2012, ApJL, 761, L23

Kato, M., \& Hachisu, I. 2003, ApJL, 598, L107

Kato, M., Hachisu, I., Kiyota, S., \& Saio, H. 2008, ApJ, 684, 1366

Kromer, M., Fink, M., Stanishev, V., et al. 2013, MNRAS, 429, 2287

Leonard, D. C., \& Filippenko, A. V. 2001, PASP, 113, 920

Li, W., Filippenko, A. V., Chornock, R., et al. 2003, PASP, 115, 453

Liu, W.-M., Chen, W.-C., Wang, B., \& Han, Z. W. 2010, A\&A, 523, A3

McClelland, C. M., Garnavich, P. M., Galbany, L., et al. 2010, ApJ, 720, 704

McCully, C., Jha, S. W., Foley, R. J., et al. 2014a, Natur, 512, 54

McCully, C., Jha, S. W., Foley, R. J., et al. 2014b, ApJ, 786, 134

Miller, J. S., \& Stone, R. P. S. 1993, Lick Obs. Tech. Rep. 66 (Santa Cruz: Lick Obs.)

Nakano, S., \& Itagaki, K. 2014, CBET, 4011, 1

Narayan, G., Foley, R. J., Berger, E., et al. 2011, ApJL, 731, L11

Ochner, P., Tomasella, L., Benetti, S., et al. 2014, ATel, 6648, 1

Phillips, M. M., Li, W., Frieman, J. A., et al. 2007, PASP, 119, 360

Pilyugin, L. S., Grebel, E. K., \& Kniazev, A. Y. 2014, AJ, 147, 131

Schoeniger, F., \& Sofue, Y. 1997, A\&A, 323, 14

Shara, M. M., Bibby, J. L., Zurek, D., et al. 2013, AJ, 146, 162

Silverman, J. M., Foley, R. J., Filippenko, A. V., et al. 2012, MNRAS, 425, 1789

Stritzinger, M. D., Hsiao, E., Valenti, S., et al. 2014a, A\&A, 561, A146

Stritzinger, M. D., Valenti, S., Hoeflich, P., et al. 2014b, A\&A, 573, A2

Valenti, S., Pastorello, A., Cappellaro, E., et al. 2009, Natur, 459, 674

Wade, R. A., \& Horne, K. 1988, ApJ, 324, 411

White, C. J., Kasliwal, M. M., Nugent, P. E., et al. 2014, arXiv:1405.7409

Woudt, P. A., Steeghs, D., Karovska, M., et al. 2009, ApJ, 706, 738 